\title{
CONOCIMIENTOS EN PROFESIONALES DE ENFERMERÍA SOBRE LESIONES NO INTENCIONALES EN LA INFANCIA TEMPRANA
}

\section{NURSING PROFESSIONAL'S KNOWLEDGE RELATED WITH UNINTENTIONAL INJURIES IN THE EARLY CHILDHOOD}

Yanet Blanco Fleites ${ }^{1}$

Julia Maricela Torres Esperón ${ }^{2}$

Ernesto Julio Bernal Valladares ${ }^{3}$

${ }^{1}$ Asesora de Enfermería de la Dirección Municipal de Salud. Municipio Cienfuegos

2 Profesora Titular. Escuela Nacional de Salud Pública. Cuba. Autor para correspondencia: maricela.torres@infomed.sld.cu

${ }^{3}$ Profesor asistente de informática médica y bioestadística. Hospital Gustavo Aldereguía Lima de Cienfuegos

\section{RESUMEN}

Introducción: Las lesiones no intencionales en la infancia temprana son un problema de salud en el mundo. Así se demuestra en el informe del 2012 de la Organización Mundial de la Salud y el Fondo de las Naciones Unidas para la Infancia explican que cada día mueren más de 2000 niños debido a lesiones no intencionales, y cada año ingresan decenas de millones con lesiones que a menudo los dejan discapacitados para toda la vida.

Objetivos: Identificar el nivel de conocimientos en enfermeros sobre lesiones no intencionales en la infancia temprana, en profesionales de enfermería y diseñar una capacitación según las necesidades de aprendizajes identificadas.

Métodos: Se realizó un estudio observacional de febrero a abril de 2016, para la identificación de conocimientos a 45 profesionales de enfermería que laboran en los consultorios médicos de la familia de los policlínicos área V, VI y VI del municipio Cienfuegos. Las necesidades de aprendizaje identificadas permitieron la elaboración y aplicación de un curso.

Resultados: De los 45 evaluados el $82.5 \%$ no alcanzan los conocimientos necesarios sobre lesiones no intencionales en la infancia temprana. Después de realizado el curso los resultados fueron satisfactorios.

Conclusiones: Se identifica la necesidad de la formación continuada en un tema 
necesario en la Atención Primaria de Salud, alternativa como las de este estudio son factibles para demostrar esta necesidad.

Palabras clave: Conocimiento, accidentes, primera infancia, enfermería

\begin{abstract}
Introduction: Unintentional early childhood injuries are a health problem in the world. This is demonstrated in the 2012 report of the World Health Organization - WHO, and the United Nations Children's Fund - UNICEF, where it is explained that more than 2000 children die every day due to unintentional injuries, and tens of millions enter each year with injuries that often leave them disabled for life.

Objectives: To identify the level of knowledge of nurses on unintentional injuries in the early childhood, in nursing professionals, and design a training program according to the identified learning needs.
\end{abstract}

Methods: An observational study was carried out from February to April 2016, for the identification of knowledge in 45 nursing professionals working at medical offices of the policlinic family areas V, VI and VII, of the Cienfuegos municipality. The learning needs identified enabled the development and implementation of a course.

Results: Of the 45 evaluated, $82.5 \%$ do not achieve the necessary knowledge related with unintentional injuries in early childhood.

After the course the results were satisfactory.

Conclusions: It was identified the need for continuing training in a necessary topic on the Primary Health Attention, alternatives as shown in this paper are feasible to demonstrate this need

Key words: Knowledge, accidents, early childhood, nursing 


\section{INTRODUCCIÓN}

El aprendizaje humano es un fenómeno complejo. En cada persona se llevan a cabo múltiples procesos cognoscitivos al momento de aprehender un nuevo conocimiento. Se trata de técnicas, inventivas, iniciativas intelectuales que enriquecen los esquemas mentales. Durante el siglo XX, diferentes paradigmas teóricos han intentado explicar, con fundamentos sólidos, cómo ocurre el aprendizaje humano. En general coinciden que el proceso de maduración cognoscitivo está asociado a una forma particular que ocurre en cada persona. Se trata pues de una manera, una forma de comportamiento intelectual dinámica, que se perfecciona durante procesos epistemológicos. ${ }^{1}$

La enfermería al igual que cualquier otra profesión, es un producto social que adopta en cada época de la historia la forma que le es posible en las circunstancias de referencias. Tiene un cuerpo de conocimientos científicos utilizados con el propósito de proporcionar cuidados a las personas de todas las edades, sanas o enfermas, en todos los contextos que incluye la promoción de salud, la prevención de las enfermedades a la familia y en general a la comunidad. ${ }^{2}$

La práctica asistencial exige que los profesionales de enfermería sean capaces de pensar críticamente para escoger las acciones idóneas para solucionar los problemas detectados. ${ }^{3}$ Las lesiones no intencionales en la infancia temprana son un problema de salud en el mundo, así se demuestra en el informe del 2012 de la Organización Mundial de la Salud (OMS) y el Fondo de las Naciones Unidas para la Infancia (UNICEF) explican que cada día mueren más de 2000 niños debido a lesiones no intencionales, y cada año ingresan decenas de millones con lesiones que a menudo los dejan discapacitados para toda la vida. Este informe, que constituye la primera evaluación mundial integral de las lesiones no intencionales en los niños, prescribe medidas para la prevención y concluye que si se adoptaran estas medidas de eficacia demostrada en todas partes podría salvarse la vida de al menos 1000 niños al día. ${ }^{4}$

En tal sentido es la primera infancia, una de las edades más vulnerables a la ocurrencia de lesiones no intencionales, en Cuba es la primera causa de muerte de este grupo de edad. ${ }^{5}$ En el caso del municipio Cienfuegos en el año 2016 ocurrieron 267 lesiones no intencionales en niños de 1 a 4 años, representando el 3.6\% de la población en este grupo de edades. ${ }^{6}$ 
Los profesionales de enfermería como parte del equipo de salud de la atención primaria, juegan un rol fundamental en la promoción de salud y prevención de lesiones como estas, tanto en las consultas de puericultura como en las visitas del hogar, establecidas en el Programa de Atención Materno Infantil (PAMI). De ahí que su preparación en temas como este, sobre todo en edades vulnerables es vital, y en tal sentido el programa nacional de prevención de accidentes para menores de 20 años ${ }^{7}$ establece objetivos y acciones al respecto, sin embargo, no establece capacitaciones frecuentes dirigidas a mejorar el desempeño de dichos profesionales.

Estos argumentos llevaron a los autores a realizar un estudio que pretende establecer una estrategia para prevención de lesiones no intencionales en la primera infancia en el Municipio Cienfuegos, parte del diagnóstico da salida a este artículo, que tiene como objetivos identificar el nivel de conocimientos en enfermeros sobre lesiones no intencionales en la infancia temprana y diseñar una capacitación según las necesidades de aprendizajes identificadas.

\section{MÉTODOLOGÍA}

Se realizó un estudio observacional con 45 profesionales de enfermería que laboran en los consultorios médicos de la familia de las áreas de salud V, VI y VII del municipio Cienfuegos. Estas áreas fueron escogidas por el criterio de frecuencia, pues eran las que tenían las cifras más elevadas de lesionados en la primera infancia en un análisis retrospectivo estadístico desde el año 2012 hasta el 2015. El período de estudio donde se aplica el instrumento abarcó los meses de febrero a Abril del año 2016.

Se diseñó y validó un instrumento con el fin de identificar el nivel de conocimientos en los profesionales de enfermería sobre lesiones no intencionales en la infancia temprana. Validado en contenido, por la metodología de criterios de expertos, estableciendo luego el índice Kappa para medir la concordancia entre los observadores. El instrumento fue aplicado a un grupo piloto, empleando el alfa de Cronbach para conocer así la consistencia interna del mismo. Quedando un instrumento fiable, válido y unidimensional con tres ejes temáticos: 1) Lesiones no intencionales en la infancia temprana, 2) Programa de prevención de accidentes para menores de 20 años, 3) Proceso de atención de Enfermería.

Las variables que se estudiaron fueron edad, categoría ocupacional, experiencia profesional y nivel de conocimientos. El nivel de conocimientos fue evaluado mediante 
un instrumento diseñado para este fin. Este último se evaluó en nota cuantitativa con clave de evaluación mediante el método criteriado.

Según los resultados y de acuerdo a la revisión bibliográfica se elaboró un curso, que fue evaluado por expertos previo a la aplicación.

Se obtuvo la autorización previa de las autoridades de salud y el consentimiento informado de todos los sujetos implicados en el estudio.

\section{RESULTADOS Y DISCUSION}

A continuación, se presentan los resultados derivados de la investigación.

Gráfico No 1: Distribución de frecuencias según edad de los enfermeros de las áreas de salud V, VI y VII. Municipio Cienfuegos. 2016.

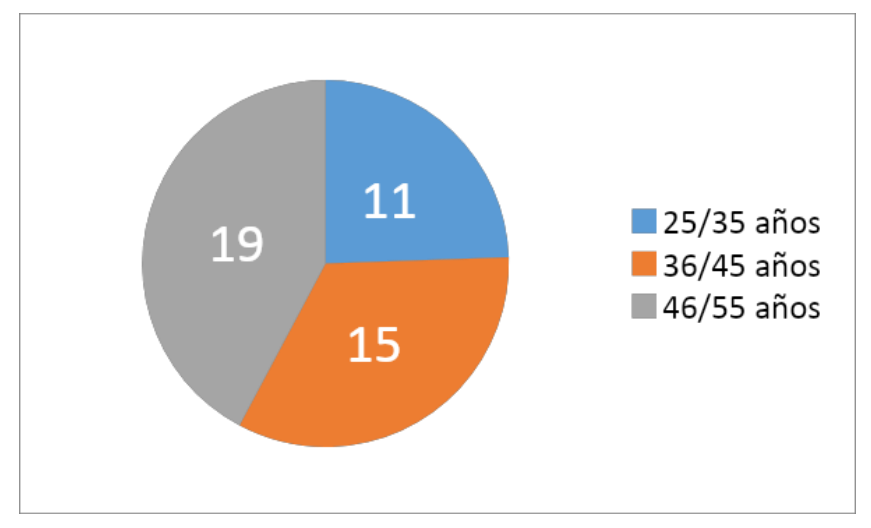

Fuente: Instrumento. Gráfico 2: Distribución de frecuencias según la categoría ocupacional de los enfermeros de las áreas de salud V, VI y VII. Municipio Cienfuegos.

2016.

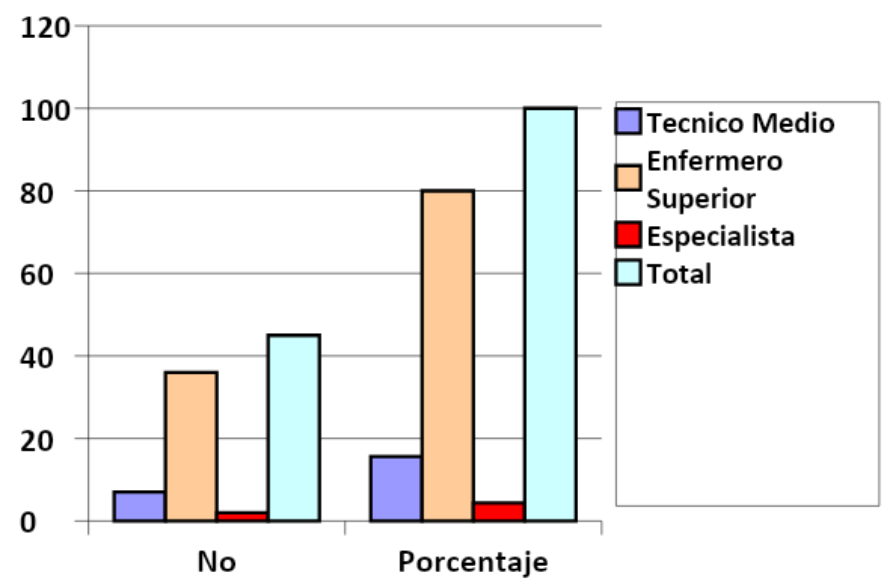

Fuente: Instrumento. 
Tabla 1: Distribución de frecuencias según experiencia profesional de los enfermeros de las áreas de salud V, VI y VII. Municipio Cienfuegos. 2016.

\begin{tabular}{|l|l|l|}
\hline Experiencia profesional & Frecuencia & Porcentaj \\
\hline De 1 año & 1 & 2.2 \\
\hline De 1 a 5 años & 10 & 22.2 \\
\hline De 6 a 10 años & 13 & 28.9 \\
\hline De 11 a 15 años & 2 & 4.4 \\
\hline De 16 a 20 años & 3 & 6.7 \\
\hline Mayor de 20 años & 16 & 35.6 \\
\hline Total & 45 & 100 \\
\hline
\end{tabular}

Fuente: Instrumento

Tabla 2: Distribución del porcentaje según el nivel de conocimientos alcanzados por los enfermeros en los tres ejes temáticos desarrollados en el instrumento en las áreas de salud V, VI, VII, Municipio Cienfuegos. 2016.

\begin{tabular}{|c|c|c|c|}
\hline Ejes temáticos & $\begin{array}{l}A-V \\
n=22\end{array}$ & $\begin{array}{l}\text { A-VI } \\
n=9\end{array}$ & $\begin{array}{ll} & \text { A } \\
\text { VII } & \\
& \\
& n=14\end{array}$ \\
\hline $\begin{array}{l}\text { Lesiones no intencionales } \\
\text { en la infancia temprana }\end{array}$ & 32,45 & 48,05 & 43,76 \\
\hline $\begin{array}{l}\text { Programa de prevención } \\
\text { de accidentes para menores de } 20 \\
\text { años }\end{array}$ & 36,52 & $59,3 \%$ & 53,54 \\
\hline $\begin{array}{l}\text { Proceso de atención de } \\
\text { Enfermería }\end{array}$ & $\%$ & 55,96 & $46,3 \%$ \\
\hline
\end{tabular}

Fuente: Instrumento.

Tabla 3: Distribución de porcentajes según el nivel de conocimientos de los enfermeros, en las preguntas desarrolladas en el instrumento en las áreas de salud V, VI, 
VII, Municipio Cienfuegos. 2016

\begin{tabular}{|c|c|c|c|c|c|c|c|c|c|c|c|c|c|c|c|c|c|c|c|c|c|}
\hline \multirow[t]{2}{*}{$\begin{array}{c}\text { Áre } \\
\mathbf{a}\end{array}$} & & & & sion & s no & ntes & iol & & & & \multicolumn{6}{|c|}{$\begin{array}{c}\text { Programa de } \\
\text { prevención de } \\
\text { accidentes }\end{array}$} & \multicolumn{5}{|c|}{$\begin{array}{l}\text { Proceso de atención de } \\
\text { enfermería }\end{array}$} \\
\hline & $\mathrm{p} 1$ & $\mathrm{p} 2$ & $\mathrm{p} 3$ & $\mathrm{p} 4$ & p5 & p6 & $p$ & & $\overline{\mathrm{p} 9}$ & $\overline{\mathrm{p} 1}$ & p11 & $\mathrm{p} 1$ & & p13 & $\mathrm{p} 12$ & $\mathrm{p} 13$ & $\mathrm{p} 16$ & $\mathrm{p} 17$ & $\mathrm{p} 18$ & p19 & $\mathrm{p} 20$ \\
\hline V & $\begin{array}{c}13 \\
6\end{array}$ & $\begin{array}{r}29 \\
5\end{array}$ & $\begin{array}{r}60 \\
9\end{array}$ & $\begin{array}{c}18 \\
6\end{array}$ & $\begin{array}{c}24, \\
7\end{array}$ & $\begin{array}{c}22, \\
7\end{array}$ & $\begin{array}{r}70 \\
4\end{array}$ & & $\begin{array}{r}61 \\
3\end{array}$ & $\begin{array}{r}18 \\
1\end{array}$ & 65 & 20 & & $\begin{array}{c}22, \\
7\end{array}$ & 4,5 & $\begin{array}{r}70 \\
4\end{array}$ & $\begin{array}{c}18 \\
1\end{array}$ & 60 & $\begin{array}{c}13, \\
6\end{array}$ & $\begin{array}{c}52 \\
7\end{array}$ & $\begin{array}{c}13, \\
6\end{array}$ \\
\hline VI & $\begin{array}{c}55 \\
5\end{array}$ & $\begin{array}{r}52 \\
7\end{array}$ & $\begin{array}{r}57 \\
7\end{array}$ & $\begin{array}{r}33 \\
3\end{array}$ & $\begin{array}{c}28, \\
8\end{array}$ & $\begin{array}{r}26, \\
6\end{array}$ & 74 & & $\begin{array}{r}91 \\
6\end{array}$ & $\begin{array}{r}35 \\
5\end{array}$ & $\begin{array}{r}71 \\
1\end{array}$ & $\begin{array}{r}53 \\
3\end{array}$ & & $\begin{array}{c}66, \\
6\end{array}$ & $\begin{array}{r}11 \\
1\end{array}$ & $\begin{array}{r}94 \\
4\end{array}$ & $\begin{array}{c}22 \\
2\end{array}$ & $\begin{array}{c}84, \\
4\end{array}$ & $\begin{array}{c}22, \\
2\end{array}$ & $\begin{array}{c}84 \\
4\end{array}$ & $\begin{array}{c}66, \\
6\end{array}$ \\
\hline VII & $\begin{array}{c}35 \\
7\end{array}$ & 41 & 60 & 15 & $\begin{array}{r}5,7 \\
1\end{array}$ & 20 & 6 & & $\begin{array}{r}19 \\
6\end{array}$ & $\begin{array}{r}37 \\
1\end{array}$ & $\begin{array}{r}57 \\
8\end{array}$ & $\begin{array}{r}45 \\
7\end{array}$ & & 50 & $\begin{array}{c}14 \\
2\end{array}$ & 10 & $\begin{array}{c}42 \\
8\end{array}$ & $\begin{array}{c}68 \\
5\end{array}$ & $\begin{array}{c}28 \\
5\end{array}$ & $\begin{array}{c}54 \\
2\end{array}$ & $\begin{array}{c}37 \\
5\end{array}$ \\
\hline
\end{tabular}

Fuente: Instrumento.

Según las necesidades de aprendizaje encontradas en la aplicación del instrumento, se diseña un curso de postgrado que le da respuestas a estas lagunas del conocimiento. El curso fue aprobado por el consejo científico de la provincia Cienfuegos en el que los profesionales de enfermería mostraron gran interés en participar.

El curso de superación se tituló "Cuidados enfermeros en la prevención y diagnóstico de las lesiones no intencionales en edades pediátricas", el mismo se concibió con 308 horas de ellas 132 presenciales y 176 no presenciales con una duración de 20 semanas. Se capacitaron 74 profesionales de enfermería que trabajaban en las áreas de salud involucradas en la investigación además del policlínico Área I (José Luis Chaviano) donde se validó el instrumento diseñado. Los temas del curso responden a los tres ejes temáticos que conformaron el instrumento creado reforzando aquellas temáticas donde mayores dificultades se identificaron. El curso fue impartido de manera simultánea en todas las áreas del municipio de Cienfuegos controlando las actividades docentes según carta metodológica No 3 del año 2009. Se realizó una evaluación final teórica práctica que evidenció la apropiación del conocimiento que tuvieron los enfermeros de los temas impartidos. 
Según los resultados de la variable edad que aparecen en el gráfico 1, el grupo de edad con mayor predominio fue la de 46-55 años para un 42,2\% del universo. Con ello se observa una alta tendencia a profesionales de experiencia, lo que se confirma con los resultados de la tabla 1 , donde se aprecia 35,6\% con más de 20 años de experiencia profesional. Lo que denota además permanencia de estos profesionales en la APS. Diferentes resultados se observaron en el estudio realizado por Miyazaki y colaboradores que la mayor parte de los profesionales $(36,3 \%)$ estaba en el intervalo de 30 a 40 años. ${ }^{8}$

Otro dato importante es la profesionalización del grupo estudio, pues el $80 \%$ es licenciado en enfermería según se puede observar en el gráfico 2, aspecto que debe valorarse tanto para los resultados obtenidos en los conocimientos, como en las posibilidades de mejora con la capacitación.

En el caso de del nivel de conocimientos según los ejes temáticos puede observarse en la tabla 2 que el eje temático del Programa de prevención de accidentes para menores de 20 años fue el de mejores resultados, dado por el uso permanente de este programa y los controles periódicos que se realizan desde los diferentes niveles municipales, provinciales y ministeriales. En segundo lugar aparece el Proceso de Atención de Enfermería diferenciado con los resultados de Martínez Isaac en su tesis doctoral en la variable número seis, que constata como muy significativo, que el $86.6 \%$ de los enfermeros demuestran en su desempeño profesional que, conoce el método científico de la profesión, pero en su aplicación presenta problemas en la autonomía y creatividad para la atención integral al paciente. ${ }^{9}$

El conocimiento acerca de las lesiones no intencionales en la infancia temprana fue el eje temático con mayores dificultades encontradas según instrumento de evaluación

En el análisis pormenorizado por preguntas que refleja la tabla 3 se observa que las preguntas $(\mathrm{P})$ con más dificultades en las tres áreas de salud son la P 14 en el eje temático 2 que aborda el tipo de prevención a utilizar y la $\mathrm{P} 18$ que examina las necesidades que pueden influir en la producción de las lesiones no intencionales en niños y niñas.

Los estilos de aprendizaje son modelos propuestos que se han generado para explicar la manera en que la persona aprende. En general, cada ser humano responde a una forma particular y predominante de aprendizaje que le permite interrelacionarse con su ambiente y que implica aspectos cognitivos referentes a la personalidad; es decir, 
características fisiológicas y psicológicas, que los diferencian unos de otros. A la hora de aprender, cada uno lo hace de manera distinta y organiza el proceso de acuerdo con la forma cómo asimilan los contenidos que estudian. ${ }^{10}$

El hecho de existir insuficiente conocimiento demuestra una debilidad del aprendizaje representando una deficiencia en la educación permanente y continuada de los servicios, ya que el conocimiento de este instrumento son requisitos casi mandatario para este personal, por estar presentes en sus procederes diarios.

Por otro lado en el modelo de formación actual del profesional de enfermería en Cuba ${ }^{11}$, se estudia en el 8vo semestre en la asignatura de Enfermería Familiar y Social las Lesiones no intencionales en la comunidad, no se encontraron otros cursos, ni formaciones de postgrados que aborden este tema para el perfeccionamiento profesional al respecto. Lo que dio lugar al diseño del curso según las necesidades de aprendizaje encontradas en el grupo de estudio.

El objetivo de aprendizaje desde hace algunos años ha variado, cambiando el modelo de enseñanza y aprendizaje tradicional de conocimientos ("saber") por el modelo actual de competencias ("saber hacer"). Uno de los objetivos del nuevo modelo es la participación del estudiante, es decir, el proceso de enseñanza--aprendizaje activo. Son numerosas las nuevas metodologías docentes que están desarrollándose, como es el Aprendizaje Basado en Problemas (ABP) con modelos de base psicológica, del método socrático, del método científico, evolucionista, el aprendizaje basado en proyectos ${ }^{12}$. El curso diseñado responde a esta metodología pues el ABP es una metodología centrada en el aprendizaje, en la investigación y reflexión que siguen los alumnos para llegar a una solución ante un problema planteado por el profesor usada en el curso de superación.

El haber identificado las preguntas con mayores dificultades en los ejes temáticos permitió diseñar una propuesta de curso que fue aprobado por el Consejo Científico de la Provincia de Cienfuegos, el cual está constituido por expertos organizados para esta funciones. Para el diseño se realizó una búsqueda, selección, registro y análisis de la información, en este sentido, se requirió de técnicas y estrategias diversas para acceder a ella, según la fuente a la que se acudió y el soporte que se utilizó, lo cual requirió el conocimiento de diversos canales y medios de comunicación (oral, impreso, audiovisual, digital o multimedia). 
En el estudio realizado por Medina González y Valcárcel Izquierdo plantean que hay que tener en cuenta el elemento motivacional para el aprendizaje, donde el profesional de Enfermería reconozca de forma objetiva sus necesidades que serán satisfechas por acciones de superación profesional y la evaluación que posibilita un enfoque de sistema, teniendo en cuenta el precepto leninista que plantea de la contemplación viva al pensamiento abstracto y de vuelta a la práctica enriquecida ${ }^{13}$.

El instrumento aplicado para medir el nivel de conocimientos sobre lesiones no intencionales en la infancia temprana abarca temas importantes del accionar de los enfermeros en la comunidad. El curso de capacitación con los resultados obtenidos refleja que los objetivos propuestos fueron alcanzados por los profesionales de enfermería.

\section{CONCLUSIONES:}

El estudio fue factible para demostrar esta necesidad de la formación continuada en un tema necesario en la Atención Primaria de Salud, aspecto que debe ser valorado para trazar estrategias de capacitación que respondan a los problemas de salud identificados.

El diseño del curso sobre la base de las necesidades de aprendizajes identificadas ofreció un producto coherente para la educación continua en un tema vulnerable y necesario en la salud infantil de estos tiempos.

\section{REFERENCIAS}

1. Bolívar López Juan M, Rojas Velázquez F. Estudio de la autopercepción y los estilos de aprendizaje como factores asociados al rendimiento académico en estudiantes universitarios. Revista de Educación a Distancia [Internet]. 2015; Disponible en: http://revistas.um.es/red/article/view/237781

2. Bello Fernández NL. Fundamentos de enfermería. Parte I. La Habana: Editorial Ciencias Médicas; 2006

3. Pereira da Silva Reichert A, Collet N, Helena Eickmann S, Carvalho Lima Maria De. Vigilancia del desarrollo infantil: estudio de intervención con enfermeros de la Estrategia Salud de la Familia. Rev. Latino-Am. Enfermagem [Internet]. 2015 [citado 27 de agosto 2017]; 23(5): 954-952 p. Disponible en: www.eerp.usp.br/rlae 
4. Peden M, Oyegbite K, Ozanne-Smith J, Hyder AA, Branche C, Rahman A, Bartolomeos K. (2012). Informe mundial sobre prevención de las lesiones en los niños. Washington, DC: $2012 . \quad$ DHO; en: http://apps.who.int/iris/bitstream/10665/77761/1/9789275316566_spa.pdf.

5. Cuba. Ministerio de Salud Pública. Anuario Estadístico del de salud. Principales causas de muerte de 1-4 años de edad. La Habana: Minsap; 2016. Disponible en: http://bvs.sld.cu/cgi-

bin/wxis/anuario/?IsisScript $=$ anuario/iah.xis\&tag5001 $=$ mostrar $^{\wedge} \mathrm{m} 1175 \& \operatorname{tag} 5009=\mathrm{ST}$ ANDARD\&tag5008=10\&tag5007=Y\&tag5003=anuario\&tag5021=e\&tag5022=2004 $\& \operatorname{tag} 5023=1175$

6. Cuba. Informe panorama del Programa de Atención Materno Infantil del Municipio de Cienfuegos. 2016. Cienfuegos: Sección Accidentes.

7. Cuba. Ministerio de Salud Pública. Programa para la prevención de accidentes en menores de 20 años. La Habana: Minsap; 1995.

8. Miyazaki MY, Caliri MH, Santos CD. Conocimiento de los profesionales de enfermería sobre prevención de la úlcera por presión. Rev. Latino-Am Enfermagem internet. 2010; 18(6): 1-9. Disponible en: http://dx.doi.org/

9. Martínez Isaac JA. Diseño por competencias del diplomado en enfermería clínicoquirúrgica [tesis]. La Habana: Universidad de Ciencias Pedagógicas "Enrique José Varona"; 2011. 10.

10. Bolívar López JM, Rojas Velásquez F. Estudio de la autopercepción y los estilos de aprendizaje como factores asociados al rendimiento académico en estudiantes universitarios. Revista de Educación a distancia. Numero 44.15 Nov-2014 http://www.um.es/ead/red/44

11. Cuba. Ministerio de Salud Pública. Plan de estudio de la carrera de Licenciatura en Enfermería. La Habana: MINSAP, 2008

12. Fidalgo Cerviño E. Conocimiento construido con la participación activa del alumno. Proyecto de Innovación y Mejora de la Calidad Docente. Madrid, España: Universidad Complutense de Madrid; 2014.

13. Medina González I, Valcárcel Izquierdo N. Superación profesional del licenciado en 
Enfermería para la solución de problemas en su desempeño profesional pedagógico.

Educ Med Super 2016; 30(1): [aprox. 11 p.]. Disponible en:

http://scielo.sld.cu/scielo.php?script=sci_arttext\&pid=S0864-

$21412016000100014 \& \operatorname{lng}=\mathrm{es}$. 